\title{
Age related axonal neuropathy in spinocerebellar ataxia type 3/Machado-Joseph disease (SCA3/MJD)
}

\begin{abstract}
Thomas Klockgether, Ludger Schöls, Michael Abele, Katrin Bürk, Helge Topka, Frank Andres, Georgios Amoiridis, Rainer Lüdtke, Olaf Riess, Franco Laccone, Johannes Dichgans
\end{abstract}

\begin{abstract}
To identify determinants of peripheral involvement in spinocerebellar ataxia type 3/Machado-Joseph disease (SCA3/MJD) the influence of CAG repeat length, age of onset, disease duration and age on the results of nerve conduction studies was analysed in 58 patients with SCA3/MJD. Patients with SCA3/MJD showed marked reduction of compound muscle action potential (CMAP) and sensory nerve action potential (SNAP) amplitudes indicating axonal neuropathy of both motor and sensory fibres. In addition, there was moderate slowing of nerve conduction suggestive of mild peripheral demyelination. Multivariate regression showed that CMAP and SNAP amplitudes decreased with age, but were not affected by CAG repeat length, age of onset, or disease duration. The age related decline of CMAP and SNAP amplitudes in SCA3/ MJD was greater than in normal subjects. The data suggest that the degree of peripheral damage in SCA3/MJD does not depend on CAG repeat length, age of onset, or disease duration, but is mainly related to the time period over which the SCA3/MJD mutation exerts its effect. (F Neurol Neurosurg Psychiatry 1999;66:222-224)
\end{abstract}

Keywords: spinocerebellar ataxia; Machado-Joseph disease; axonal neuropathy; CAG repeat mutation

Department of Molecular Human Genetics, University of Bochum, Bochum, Germany

O Riess

Department of Human Genetics, University of Göttingen, Göttingen. Germany

F Laccone

Correspondence to: Dr T Klockgether, Department of Neurology, University of Bonn, Sigmund-Freud-Str. 25, D-53105 Bonn,

Germany.

Received 17 April 1998 Accepted 24 July 1998

Spinocerebellar ataxia type 3/Machado-Joseph disease (SCA3/MJD) is an autosomal dominant inherited neurodegenerative disorder with a wide range of clinical manifestations including ataxia, ophthalmoplegia, pyramidal signs, basal ganglia symptoms, and peripheral neuropathy. ${ }^{1-3}$ The mutation causing SCA3/ MJD has been shown to be an unstable CAG trinucleotide repeat expansion within the coding region of a gene of unknown function that is located on chromosome $14 \mathrm{q} .{ }^{4}$ Whereas the repeat length in normal subjects varies between 14 and 40 CAG units, patients with SCA3/ MJD have one allele within a range of 62 to 84 units. As in other CAG repeat disorders, there is an inverse correlation between repeat length and age of onset in SCA3/MJD with the largest alleles occurring in patients with early onset of disease and smaller alleles in patients with late onset of disease. ${ }^{4}$

Clinical symptoms of peripheral neuropathy with sensory disturbances, areflexia, weakness, and amyotrophy are more frequent in patients with SCA3/MJD with late disease onset suggesting a paradoxical influence of CAG repeat length with stronger involvement in patients with smaller expansions and later disease onset. ${ }^{5-8}$ Alternatively, the degree of peripheral involvement may not be related to CAG repeat length, but rather to age with greater frequency of neuropathy in older patients with SCA3/MJD. To identify determinants of peripheral involvement in SCA3/MJD we analysed the influence of CAG repeat length, age of onset, disease duration, and age on the results of nerve conduction studies in 58 consecutive patients with SCA3/MJD.

\section{Methods}

A diagnosis of SCA3 was made in 58 patients (31 female, 27 male) by demonstration of CAG expansion at the SCA3 locus using polymerase chain reaction as described in detail elsewhere. $^{347}$ CAG repeat length of the expanded alleles ranged from 63 to 82 with a mean of 73.6 (SD 4.0). Mean age was 46.8 (SD 12.1) years, mean age of onset 37.8 (SD 11.3) years, and mean disease duration 9.1 (SD 5.9) years. A group of 91 healthy volunteers ( 47 female, 44 male; mean age: 45.9 

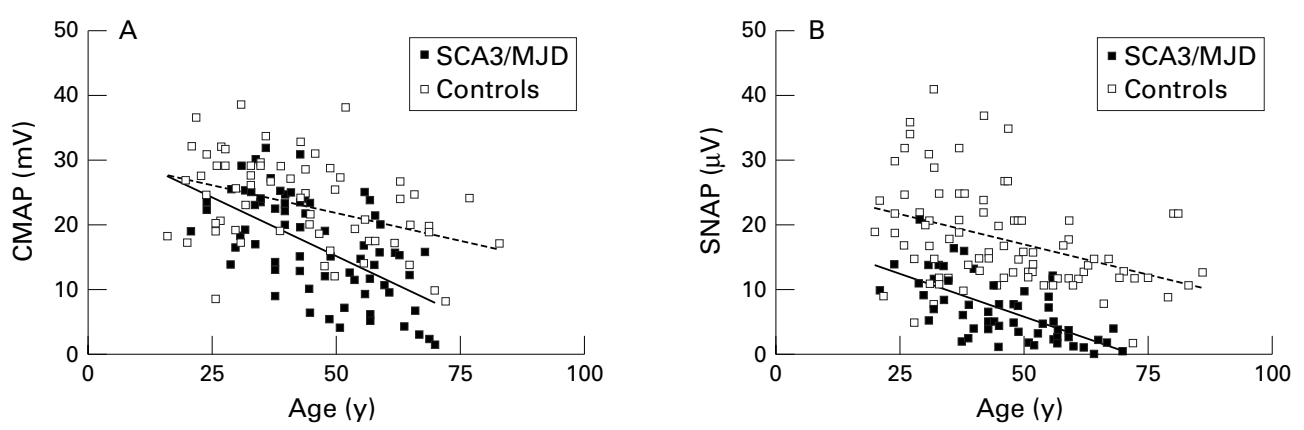

Correlation between (A) CMAP and (B) SNAP amplitude and age. Each plot gives the data of patients with $S C A 3 / M F D$ (closed symbols, uninterrupted line) and controls (open symbols, dashed line).

All patients underwent nerve conduction studies using standard neurographic procedures. Motor nerve conduction studies were performed on the tibial nerve of the left leg. Sensory nerve conduction velocities of the sural nerve of the left leg were measured antidromically. Amplitudes of the evoked motor (abductor hallucis muscle) and sensory responses were measured with surface electrodes. Skin temperature was routinely controlled, and the limbs were warmed if necessary.

Electrodiagnostic variables used for regression analysis were motor nerve conduction velocity (m-NCV), distal motor latency (DL), amplitude of compound muscle action potential (CMAP), sensory nerve conduction velocity (s-NCV), and amplitude of the sensory nerve action potential (SNAP).

\section{Results}

The NCVs of patients with SCA3/MJD were normal or moderately reduced. Mean m-NCV was 45.1 (SD 4.4) $\mathrm{m} / \mathrm{s}$ (controls: 46.7 (SD 3.0 $\mathrm{m} / \mathrm{s}$ ) and mean s-NCV 44.7 (SD 5.2 ) $\mathrm{m} / \mathrm{s}$ (controls: 49.0 (SD) $4.1 \mathrm{~m} / \mathrm{s}$ ). Mean DL was slightly prolonged (4.4 (SD 1.1) v 3.9 (SD 0.5) $\mathrm{ms})$. CMAP and SNAP amplitudes were markedly reduced in SCA3/MJD. Mean CMAP amplitude was 16.4 (SD 7.6) $\mathrm{mV}$ (controls: 23.0 (SD 6.9) mV), and mean SNAP amplitude 6.7 (SD 4.7) $\mu \mathrm{V}$ (controls: 17.8 (SD 7.5) $\mu \mathrm{V})$ (table).

Simple linear regressions with Bonferroni adjustment disclosed significant negative correlations of CMAP and SNAP amplitudes with age $(p=0.0020, p=0.0017)$ and age of onset $(\mathrm{p}=0.0032, \mathrm{p}=0.0018)$, as well as CMAP amplitudes with disease duration $(p=0.0195)$. By contrast, CMAP and SNAP amplitudes were positively correlated with CAG repeat length $(p=0.0392, p=0.0019)$. There were no significant correlations of $\mathrm{m}-\mathrm{NCV}$, DL, or s-NCV with CAG repeat length, age of onset, disease duration, and age. Therefore, only the CMAP and SNAP data were further analysed using multivariate regression.

To select the appropriate statistical model, we used a stepwise regression procedure based on the Akaike information criterion. As a result of this procedure, we chose a statistical model for analysis of both CMAP and SNAP data that included age as the only covariable. Addition of any other covariable did not yield a better fit. Regression analysis showed that age was nega- tively correlated with both CMAP $(\mathrm{p}<0.0001$, $r=-0.59)$ and SNAP amplitude $(\mathrm{p}<0.0001$, $r=-0.69$ ) (figure). The annual decline of the CMAP amplitude was significantly faster in SCA3/MJD (0.36 mV/year) than in controls $(0.17 \mathrm{mV} /$ year; $\mathrm{p}=0.0146, t$ test within analysis of variance (ANOVA) with age-group interaction). Similarly, SNAP amplitude declined faster in patients $(0.26 \mu \mathrm{V} /$ year $)$ than in controls $(0.18 \mu \mathrm{V} /$ year $)$. However, this difference failed to reach significance. Whereas the CMAP y intercept, which is a hypothetical value of the amplitude at age of 0 years, was similar in SCA3/MJD patients $(33.4 \mathrm{mV})$ and controls $(30.3 \mathrm{mV})$, the SNAP y intercept was significantly smaller in patients $(19.0 \mu \mathrm{V})$ than in controls $(26.3 \mu \mathrm{V}, \mathrm{p}=0.0484)$.

\section{Discussion}

The present nerve conduction data show that SCA3/MJD is associated with marked reduction of CMAP and SNAP amplitudes indicating axonal neuropathy of both motor and sensory fibres. In addition, there was moderate slowing of nerve conduction suggestive of mild peripheral demyelination. Whereas the degree of nerve conduction slowing was not significantly related to CAG repeat length, age of onset, disease duration, and age, CMAP and SNAP amplitudes decreased with age but were not additionally affected by CAG repeat length, age of onset, and disease duration.

The present results agree with those of earlier nerve conduction studies in SCA3/MJD showing marked attenuation of CMAP and SNAP amplitudes and mild reduction of NCVs. ${ }^{89}$ The neuropathological basis of these abnormalities is loss of myelinated and unmyelinated fibres with an increase in endoneurial collagen. ${ }^{10}$ Although not explicitly shown for SCA3/MJD, CMAP and SNAP amplitudes may be taken as an indirect measure of the number of peripheral motor and sensory axons.

Our analysis shows that age is the most important determinant of the severity of axonal loss in SCA3/MJD. Loss of peripheral axons is a normal phenomenon occurring with age. ${ }^{11}$ In patients with SCA3/MJD, however, the age related decline of CMAP and less so of SNAP amplitudes was faster than in normal subjects suggesting that the mutated SCA3/MJD gene product accelerates the normal age related loss of peripheral axons. In addition, our data sug- 
gest that SNAP amplitudes are themselves smaller in patients than in normal subjects.

Clinical evidence suggests that many aspects of the SCA3/MJD phenotype are directly influenced by CAG repeat length. Patients with longer CAG repeat expansions are more severely affected, have earlier disease onset, faster progression, and more frequent involvement of the basal ganglia and pyramidal tract. ${ }^{16712}$ It was therefore of interest to study the influence of CAG repeat length on the loss of peripheral axons. Initial analysis with simple linear regression suggested a paradoxical association of small CAG repeat expansions with more severe axonal neuropathy. However, subsequent analysis using multivariate methods disclosed that this association is an artefact explained by the colinearity of CAG repeat length and age. We were unable to demonstrate an effect of CAG repeat length on CMAP and SNAP amplitudes.

Our data suggest that the mechanisms underlying the loss of peripheral axons in SCA3/MJD are different from those leading to degeneration of central neurons. Whereas the velocity and extent of the CNS degeneration seem to depend on CAG repeat length, the severity of peripheral damage is mainly related to the time period over which the SCA3/MJD mutation exerts its effect. There is no evidence that there is an additional effect of CAG repeat length on the loss of peripheral axons.

1 Matilla T, McCall A, Subramony SH, et al. Molecular and clinical correlations in spinocerebellar ataxia type 3 and Machado-Joseph disease. Ann Neurol 1995;38:68-72.

2 Rosenberg RN. Machado-Joseph disease: an autosomal dominant motor system degeneration. Mov Disord 1992;7: 193-203.

3 Bürk K, Abele M, Fetter M, et al. Autosomal dominant cerebellar ataxia type I: clinical features and MRI in families ebellar ataxia type I: clinical features and MRI in families
with SCA1, SCA2 and SCA3. Brain 1996;119:1497-505.

4 Kawaguchi Y, Okamoto T, Taniwaki M, et al. CAG expansions in a novel gene for Machado-Joseph disease at chromosome 14q32.1. Nat Genet 1994;8:221-8.

5 Coutinho P, Andrade C. Autosomal dominant system degeneration in Portuguese families of the Azores islands. A new genetic disorder involving cerebellar, pyramidal, extrapyramidal and spinal cord motor functions. Neurology 1978;28:703-9.

6 Maciel P, Gaspar C, DeStefano AL, et al. Correlation between CAG repeat length and clinical features in

7 Schöls L, Amoiridis G, Epplen JT, et al. Relations between genotype and phenotype in German patients with the genotype and phenotype in German patients with the Machado-Joseph disease
chiatry 1996;61:466-70.

8 Colding Jorgensen E, Sorensen SA, Hasholt L, et al. Electrophysiological findings in a Danish family with Machado-Joseph disease. Muscle Nerve 1996;19:743-50.

9 Abele M, Bürk K, Andres F, et al. Autosomal dominant cerebellar ataxia type I. Nerve conduction and evoked potential studies in families with SCA1, SCA2, and SCA3. Brain 1997;120:2141-8.

10 Coutinho P, Guimaraes A, Pires MM, et al. The peripheral neuropathy in Machado-Joseph disease. Acta Neuropathol (Berl) 1986;71:119-24.

11 Taylor PK. Non-linear effects of age on nerve conduction in adults. I Neurol Sci 1984;66:223-34.

12 Klockgether T, Kramer B, Lüdtke R, et al. Repeat length and disease progression in spinocerebellar ataxia type 3 . Lancet 1996;348:830. 